\title{
Functional Imaging of the Visual Cortex With Bold-Contrast MRI: Hyperventilation Decreases Signal Response
}

\author{
M. Weckesser, ${ }^{1,2^{*}}$ S. Posse, ${ }^{1}$ U. Olthoff, ${ }^{1}$ L. Kemna, ${ }^{1,2}$ S. Dager, ${ }^{3}$ \\ and H.-W. Müller-Gärtner ${ }^{1,2}$
}

Hypocapnia due to hyperventilation reduces cerebral blood flow and volume. To investigate the effects of hyperventilation on the regional signal response to visual activation using blood oxygenation level-dependent (BOLD) magnetic resonance imaging (MRI), six volunteers were investigated during visual stimulation under normocapnia and hypocapnia conditions. Hyperventilation significantly decreased in visual cortex the BOLD MRI response to visual stimulation $(3.97 \pm 0.5 \%$ [mean (SD) in normocapnia vs. $0.77 \pm 0.7 \%$ in hypocapnia, $P<0.01]$. In three of six subjects, functional signal changes were reduced to noise level. The reduced stimulus response during hyperventilation is probably due to a decreased overshoot in the blood oxygenation response. These results indicate that BOLD-contrast functional MRI is highly sensitive to $\mathrm{pCO}$ changes. Magn Reson Med 41:213-216, 1999. 1999 Wiley-Liss, Inc.

Key words: hyperventilation; BOLD-contrast; visual activation; fMRI

Regional increases in oxygen demand and cerebral blood flow occur during task-related neuronal activity. Increased tissue perfusion, which enlarges the available oxygen supply, exceeds the increase in regional oxygen consumption. As a consequence, there is a greater proportion of oxyhemoglobin in the capillary and venous beds of activated brain areas. Since regional blood volume changes only a small amount, a net reduction in paramagnetic deoxyhemoglobin occurs, which increases the signal intensities in susceptibility-sensitive functional magnetic resonance imaging (fMRl). This method, referred to as blood oxygenation level-dependent (BOLD) MRI, is increasingly used for mapping human brain function $(1-3)$.

Global cerebral blood flow is primarily regulated by the arterial $\mathrm{pCO}_{2}$ (4). The interaction of regional and global perfusion has been investigated by positron emission tomography (PET) $(5,6)$, but the effect of hypocapnia on task-related functional signal changes by BOLD MRI has not yet been addressed. Such studies are of interest as previous investigations have demonstrated that hyperventilation strongly reduces global cortical MR signal intensity $(7,8)$, which is thought to reflect increased oxygen extraction due to cerebral vasoconstriction and decreased cerebral blood flow. Additionally, hypocapnia reduces regional cerebral blood volume (9). In baboons, Archer et al (10)

'Institute of Medicine, Research Center Jülich, 52426 Jülich, Germany. 2Department of Nuclear Medicine, Heinrich Heine University, Düsseldorf, Germany. ${ }^{3}$ Departments of Psychiatry and Behavioral Sciences and Bioengineering, University of Washington, Seattle, Washington

'Correspondence to: M. Weckesser, Institute of Medicine, Research Center Jülich, 52426 Jülich, Germany. E-mail: m.weckesser(fz-juelich.de Received 12 February 1998; revised 30 June 1998; accepted 1 July 1998. D 1999 Wiley-Liss, Inc. described a $16 \%$ decrease in cerebral blood volume and a $30 \%$ decrease in blood flow when $\mathrm{pCO}^{2}$ was reduced from 40 to $25 \mathrm{mmHg}$. The oxygen extraction increases and during prolonged hypocapnia an increase in lactate can be measured (11). The BOLD signal is not only dependent on perfusion but is also modulated by cerebral blood volume and by the oxygen extraction fraction. Differences of functional PET and BOLD fMRI are thus likely. Due to the partly antagonistic actions of neural activation and hyperventilation, it is difficult to predict the effect of hyperventilation on the functional BOLD signal.

The aim of the present study was to investigate the specific effect of hyperventilation on the amplitude of regional BOLD fMRI signal intensity change during visual activation.

\section{MATERIALS AND METHODS}

\section{MR Studies}

A Siemens Vision 1.5 T whole body scanner (Siemens Medical Systems, Erlangen, Germany) equipped with a standard quadrature head coil was used to study six healthy young volunteers (five male and one female; aged $31.2 \pm 3.3$ years ). Written informed consent was obtained from all subjects in a manner approved by the Ethics Committee of the University of Düsseldorf.

The subjects were positioned supine in the magnet with the head fixed using a vacuum cushion and tape to avoid motion. All subjects had had previous experience with fMRI, and hyperventilation was practiced prior to the study to minimize head motion. A plastic tube was placed in the left nostril to withdraw expiratory air for continuous $\mathrm{pCO}_{2}$ monitoring (Datex Capnomac monitor). A home-built red LED array with a flicker rate of $8 \mathrm{~Hz}$ was positioned in front of the subjects' eyes. The flicker rate and timing to turn on the LED array were computer controlled and synchronized to the image acquisition of the MR scanner. Sagittal, transaxial, and coronal scout images were used to position the head in the center of the scanner. Four adjacent $6 \mathrm{~mm}$ thick slices were positioned parallel to the calcarine fissure as identified on the sagittal image. The lowest slice was located superior to the cerebellar vermis. BOLD images were acquired using echoplanar imaging (TR $1 \mathrm{sec}, \mathrm{TE} 66 \mathrm{msec}$, flip angle $30^{\circ}$ ) to measure 100-125 time frames. Three different experiments were carried out sequentially during a single imaging session: 1. During normocapnia, $30 \mathrm{sec}$ of baseline measurements were followed by three repetitions of a 10-sec visual stimulation and a 20 -sec rest period and finally by $5 \mathrm{sec}$ of visual activation. 2. Following 40 baseline measurements, sub213 
jects were asked to hyperventilate vigorously without moving their heads while 60 image data sets were sequentially acquired; 3 . Immediately following experiment 2 , subjects were asked to maintain hyperventilation while the visual activation paradigm, as described in experiment 1 , was applied.

For one of the subjects, an additional experiment was performed using a longer repetition time $(3 \mathrm{sec})$ and a larger flip angle $\left(90^{\circ}\right)$ to combine the above-mentioned three experiments into a single measurement. This permitted continuous monitoring of the cortical baseline signal and visually evoked signal changes during normo- and hypocapnia. After a 30 -sec baseline period, visual activation for $12 \mathrm{sec}$ and rest periods of $21 \mathrm{sec}$ were repeated three times. After the third rest period, hyperventilation was started and maintained during visual activation using the same timing as applied during normocapnia.

\section{Data Analysis}

All images were transferred to a SPARC 20 workstation for image analysis using an IDL (Boulder, Colorado) based DPA-software package, developed by Goldberg and LeBihan (12). A cine loop visualization of the time series was used to detect head motion. At this stage, data from one additional volunteer were discarded from further analysis due to head motion. Furthermore, the first six images of all measurements, which were acquired during non-steadystate conditions, were discarded.

Functional signal changes in the images of experiment 1 were detected using a correlation analysis with a reference function, modeling the ideal signal response. Pixels with a correlation coefficient above 0.6 were considered significantly activated by the visual stimulation. The resulting calculated images were carefully examined for motion artifacts, which can show up as false activation around the perimeter of the brain and in the ventricles. Regions of interest (ROIs) were drawn for the visual cortex using the normocapnic light-activated BOLD images from two adjacent slices. An additional ROI was created in the anterior part of the lateral temporal cortex, which was not activated by the visual paradigm. This region was employed to determine the noise level. The ROIs identified during normocapnia were used for the analysis of all protocols. The amplitude of the signal changes in the ROIs was calculated by subtracting the last three image intensity values of each rest period from the last three image intensity values of the corresponding activation period. All signal change amplitudes were expressed as a percentage of the initial baseline value. Mean signal changes during normocapnia were compared with the mean signal changes during hypocapnia in each individual. The statistical significance of differences between the two conditions was tested with a paired t-test. An error probability $P<0.05$ was considered to be statistically significant. Data are presented as mean \pm standard deviation.

In addition, a standardized statistical analysis [SPM96 softivare (13)] was used to determine the extent of activation during normo- and hypocapnia. For this purpose, a delayed box-car analysis was used. Significance thresholds of $P=0.001$ for a single voxel and $P=0.01$ for the size of a cluster were chosen. Statistical analysis was done both in the original data sets and after applying the algorithm for motion correction included in the SPM96 software. The number of activated voxels in the visual cortex was determined for the two conditions.

The time course of experiment 2 was analyzed using a curve fitting algorithm. This algorithm assumes a steady signal during the rest condition and an exponential decay during hyperventilation. The curve is expected to approach a steady state. As described in detail elsewhere (8), the fitting algorithm calculates automatically the level of the baseline signal, the time point of initial signal decrease, a time constant of the exponential function, and the signal level during the steady state of hyperventilation.

\section{RESULTS}

Hyperventilation reduced the baseline signal intensity in the visual cortex by $5.4 \pm 1.6 \%$. The BOLD responses to visual stimulation during normo- and hypocapnia are summarized in Table 1. End-tidal $\mathrm{pCO}_{2}$ decreased from $40.4 \pm 1 \mathrm{mmHg}$ during normocapnia to $18.2 \pm 3.5 \mathrm{mmHg}$ at steady state during sustained hyperventilation. Visual stimulation increased signal intensity by $3.97 \pm 0.5 \%$ during normocapnia. For the same regions, hyperventilation significantly decreased the effect of visual stimulation (from $3.97 \pm 0.5 \%$ to $0.77 \pm 0.7 \%, n=6, P<0.01$, two-tailed paired t-test). For three of the six subjects, the signal response to the visual stimulus was reduced to noise level during hypocapnia. The noise level, determined as

Table 1

Summary of the Results of All Subjects*

\begin{tabular}{|c|c|c|c|c|c|c|c|c|}
\hline \multirow{2}{*}{$\begin{array}{c}\text { Subject } \\
\text { no. }\end{array}$} & \multicolumn{2}{|c|}{$\mathrm{pCO}_{2}(\mathrm{mmHg})$} & \multicolumn{2}{|c|}{ Activation amplitude (\%) } & \multicolumn{2}{|c|}{ Noise levela $(\%)$} & \multicolumn{2}{|c|}{ No. of activated voxels (SPM96) } \\
\hline & Normocapnia & Hypocapnia & Normocapnia & Hypocapnia & Normocapnia & Hypocapnia & Normocapnia & Hypocapnia \\
\hline 1 & 39.0 & 19.4 & 4.05 & 0.52 & -0.13 & -0.13 & 127 & 0 \\
\hline 2 & 41.7 & 12.9 & 4.14 & -0.08 & -0.45 & -1.97 & 141 & 0 \\
\hline 3 & 41.0 & 20.8 & 3.84 & 0.13 & -0.54 & 0.10 & 129 & 0 \\
\hline 4 & 40.0 & 22.6 & 3.10 & 1.22 & 0.01 & -0.12 & 135 & 0 \\
\hline 5 & 41.0 & 16.0 & 4.58 & 1.24 & 0.25 & -0.31 & 184 & 0 \\
\hline 6 & 40.0 & 17.7 & 4.09 & 1.58 & -0.28 & -0.17 & 194 & 0 \\
\hline Mean & 40.4 & 18.2 & 3.97 & 0.77 & -0.19 & -0.43 & 152 & 0 \\
\hline SD & 1 & 3.5 & 0.5 & 0.7 & 0.3 & 0.76 & 30 & 0 \\
\hline
\end{tabular}

*The columns contain subject number, end-expiratory $\mathrm{pCO}_{2}$ during normo- and hypocapnia, activation amplitude during normo- and hypocapnia, noise level during normo- and hypocapnia, and number of activated voxels as identified by an SPM96 analysis.

aDefined as "activation amplitude" in a temporal lobe region that is not activated by visual stimulation. 
FIG. 1. Results of the pixel-based analysis of the effect of visual stimulation during normocapnia (left) and hypocapnia (right). All pixels shown in white exhibit a positive correlation to the reference function (correlation coefficient $>0.6$ ). Four adjacent axial echoplanar images through the occipital cortex are shown for each condition. An extensive activation of primary and secondary visual areas is evident in normocapnia; no significant activation remains during hypocapnia.

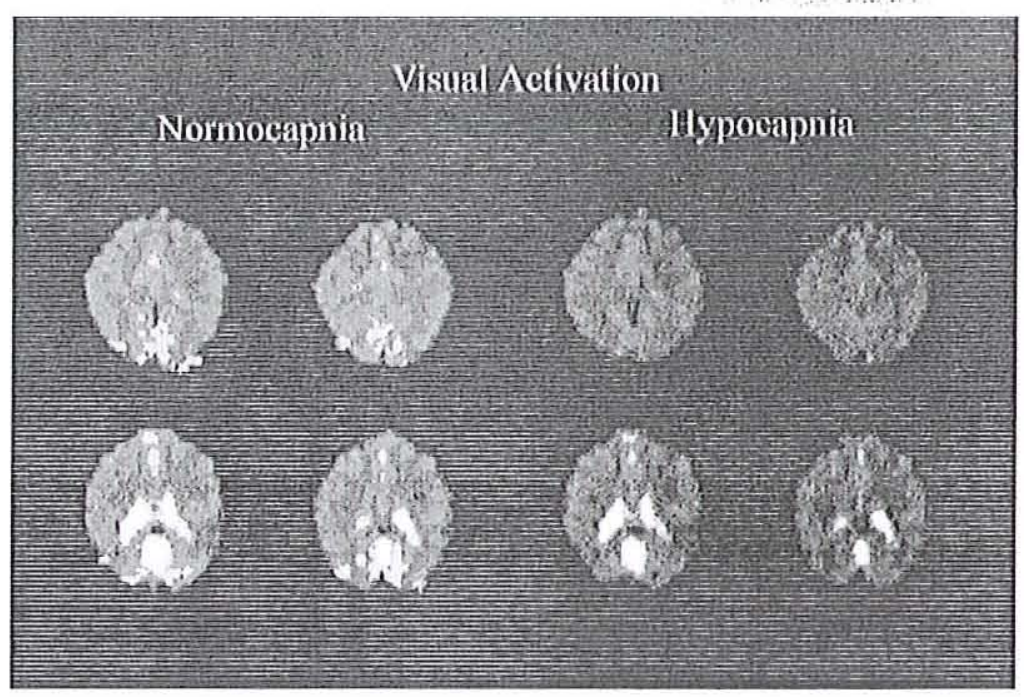

"activation amplitude" in the reference region changed insignificantly from $-0.19 \pm 0.30 \%$ during normocapnia to $-0.43 \pm 0.76 \%$ during hyperventilation (n.s., $n=6, P=0.5$, two-tailed paired t-test). The SPM96 analysis of the original data sets depicted an average of $152 \pm 30$ voxels visually activated during normocapnia, while no voxels were activated above threshold during hypocapnia. After motion correction, the number of voxels activated during normocapnia decreased slightly to $131 \pm 45$, and again no activated voxels were found during hypocapnia. In four of the volunteers, motion correction of the normocapnia condition resulted in frontal artifacts in the statistical images.

An example of the results of the pixel based correlation analysis is shown in Fig. 1. During normocapnia large parts of primary and secondary visual cortex are activated by the intense visual stimulus. During hyperventilation, no pixels with a correlation coefficient above 0.6 are identified in this case.

No studies included in this analysis showed artifacts due to head motion, as described in the methods section.

For the additional experiment using longer repetition times, these findings were replicated (Fig. 2). The baseline decrease after initiation of hyperventilation is consistent with the baseline reduction in experiment 2 and with our previous observations (8). The complete loss of activation response is representative of our findings in experiment 3 for three subjects.

\section{DISCUSSION}

Our findings demonstrate that hypocapnia reduces the task-related regional BOLD-contrast response. The BOLD signal is modulated by a number of physiological parameters, which are altered both by hyperventilation and during neuronal activation. The interaction of the various parameters may explain the loss of the BOLD MRI response. As shown by PET studies, hyperventilation decreases the perfusion response to visual stimulation. In these studies, relative perfusion changes remained constant, since baseline perfusion was reduced to the same extent as the activation amplitude $(5,6)$. As described above, BOLD MRI signal depends on an overshoot of perfusion response and an increase in oxyhemoglobin concentration during neuronal activation. During hypocapnia the perfusion response is decreased by the same amount as baseline blood flow. Therefore the overshoot of perfusion increase may be abolished, and the task-related increase in oxyhemoglobin concentration may be reduced. Furthermore, the activation-induced increase in cerebral blood volume, which leads to an increase of paramagnetic deoxyhemoglobin and thus to a signal decrease, may gain additional weight by the increased overall concentration of deoxyhemoglobin $(7,8)$. During normocapnia, this increase in blood volume is not sufficient to counterbalance the

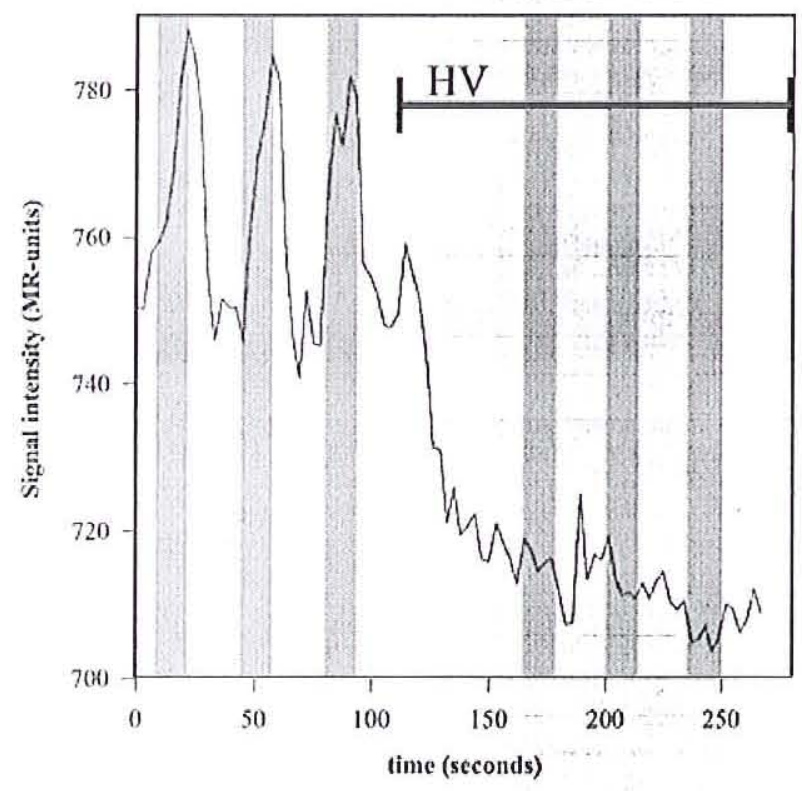

FIG. 2. Time course of signal intensity in the experiment with a long repetition time $(3 \mathrm{sec})$, displaying visual activation during normocapnia and the effect of hyperventilation and visual activation during hypocapnia [visual stimulation indicated by the gray background; hyperventilation (HV) indicated by the fat horizontal line]. 
decrease of the venous deoxyhemoglobin fraction. It is further possible that hyperventilation may alter neuronal stimulus-evoked activity (14).

The above-mentioned physiological considerations explain the reduction of the regional stimulation-induced BOLD response during hypocapnia. Furthermore, the experiment with the longer repetition time indicates that the results are reproducible. However, putative methodological limitations need to be discussed. Additional loss of sensitivity may arise from the decreased baseline signal intensity, which corresponds to a reduced $\mathrm{T}_{2}{ }^{*}$ value. For example, a $10 \%$ reduction in baseline fMRI signal corresponds to a $10 \%$ reduction in $\mathrm{T}_{2}{ }^{*}$ (assuming a monoexponential decay) (15). Since optimum sensitivity with BOLDcontrast fMRI is achieved when TE is equal to $\mathrm{T}_{2}{ }^{*}$, it may be necessary to reduce TE during hypocapnia to maintain sensitivity. However, this effect is small and is not sufficient to explain the nearly complete loss in functional signal contrast observed in some subjects. Head motion during hyperventilation could have obscured the signal response to visual stimulation. However, careful preparation of the volunteers minimized head motion, which was confirmed by inspection of the images. All image sets were assessed for motion artifacts both in the raw images and in the calculated images. As the regions chosen were relatively large and averaged over two slices, any motion not detected by visual inspection should have caused only minor effects. Motion correction did not increase the number of activated voxels, but due to the small number of slices, the reliability of motion correction is limited.

The effects of lowered end-tidal $\mathrm{CO}_{2}$ on BOLD MRI contrast reported in this study are applicable to other BOLD MRI functional imaging protocols. However, the respiratory challenge applied was extremely strong and the effects of smaller alterations in $\mathrm{pCO}_{2}$ remain to be investigated. Due to the complexity of the physiological changes that may influence the BOLD signal, results may be different when $\mathrm{pCO}_{2}$ is modified only slightly. Furthermore, the physiological responses during neuronal activity may be dynamic and may change during sustained activation (16).

The differential effects of emotional stimulation on respiration are not known at the present time. Besides the potential stress of being placed inside a dark and narrow scanner tunnel, some activating stimuli may provoke hyperventilation. Thus, the magnet environment and activation paradigms involving, for example, emotional material, may influence the respiration rate and consequently BOLD contrast, particularly among anxious subjects.

\section{REFERENCES}

1. Le Bihan D, Karni A. Applications of magnetic resonance iniaging to the study of human brain function. Curr Opin Neurobiol 1995:5:231-237.

2. Kwong KK. Functional magnetic resonance imaging with echo planar imaging. Magn Reson Q 1995:11:1-20.

3. Turner R. Magnetic resonance imaging of brain function. Am J Physiol Imaging 1992:3/4:136-145.

4. Kety S, Schmidt CF. The effect of altered arterial tensions of carbon dioxide and oxygen on cerebral blood flow and cerebral oxygen consumption in normal young men. J Clin Invest 1948;25:107-119.

5. Ramsay SC, Murphy K, Shea SA, Friston KJ, Lammertsma AA, Clark JC, Adams L, Guz A, Frackowiak RSJ. Changes in global cerebral blood flow in humans: effect on regional cerebral blood flow during a neural activation task. J Physiol 1993;471:521-534.

6. Shimosegawa E, Kanno I, Hatazawa J, Fujita H, lida H, Miura S, Murakami $\mathrm{M}$, Inugami A, Ogawa T, Itoh $\mathrm{H}$, Okudera $\mathrm{T}$, Uemura $\mathrm{K}$. Photic simulation study of changing arterial partial pressure level of carbon dioxide. J Cereb Blood Flow Metab 1995;15:111-114.

7. Stehling MK. Schmitt F, Ladebeck R. Echo-planar imaging of human brain oxygenation changes. J Magn Reson Imaging 1993:3:471-474.

8. Posse S, Olthoff U, Weckesser M, Jäncke L, Müller-Gärtner HW, Dager $\mathrm{SR}$. Regional dynamic signal changes during initiation of controlled hyperventilation assessed by BOLD-contrast RMRI. Am J Neuroradiol 1997; 18:1763-1770.

9. Grubb RL. Raichle ME, Eichling JO, Ter-Pogossian MM. The effects of changes in $\mathrm{PaCO}_{2}$ on cerebral blood volume, blood flow and vascular mean transit time. Stroke 1974;5:630-639.

10. Archer DP, Labrecque P, Tyler JL, Meyer E, Evans AC, Villemure JG, Casey WF, Diksic M, Hakim AM, Trop D. Measurement of cerebral blood flow and volume with positron emission tomography during isoflurane administration in the hypocapnic baboon. Anesthesiology 1990;72: 1031-1037.

11. Posse S, Dager SR, Richards TL, Yuan C, Ogg R, Artru AA, MüllerGärtner HW. Hayes C. In vivo measurement of regional brain metabolic response to hyperventilation using magnetic resonance: proton echo planar spectroscopic imaging (PEPSI). Magn Reson Med 1997;37:858-865.

12. LeBihan D. Integrated processing software for functional MRI. Human Brain Mapping 1995:Suppl 1:151.

13. Friston KJ, Holmes AP, Worsley KJ, Poline JB, Frith CD, Frackowiak RSJ. Statistical parametric mapping in functional imaging: a general linear approach. Hum Brain Mapping 1995;2:189-210.

14. van Rijen PC, Luyten PR, Berkelbach van der Sprenkel JW, Kraaier V, van Huffelen AC, Tulleken CAF, den Hollander JA. 'H and 3iP NMR measurement of cerebral lactate, high energy phosphate levels, and $\mathrm{pH}$ in humans during voluntary hyperventilation: associated EEG, capnographic and Doppler findings. Magn Reson Med 1989;10:182-193.

15. Gati JS, Menon RS, Ugurbil K, Rutt BK. Experimental determination of the BOLD field strength dependence in vessels and tissue. Magn Reson Med 1997:38:296-302.

16. Hathout GM, Gambhir SS, Gopi RK, Kirlew KAT, Chol Y, So G, Gozal D. Harper R, Lufkin RB, Hawkins R. A quantitative physiologic model of blood oxygenation for functional magnetic resonance imaging. Invest Radiol 1995:11:669-682.

\section{Announcements of Meetings}

The 2nd International Workshop on Hyperpolarized Gases in Magnetic Resonance: Biomedical Investigations and Clinical Applications will be held June 21 - 25, 1999 at the 'Centre de Physique' at Les Houches, France. For information please contact Dr. Genevieve Tastevin, Laboratoire Kastler Brossel, Department de Physique de l'E.N.S., 24 rue Lhomond; 75231 Paris Cedex 05, France, tel.: 33-1-44-32-20-25, fax: 33-1-45-35-00-76, e-mail: tastevin@physique.ens.fr; or Dr. Hans-Ulrich Kauczor, Klinik fuer Radiologie, Universitaet Mainz, Langenbeckstr.1; 55131 Mainz, Germany, tel.: 49-6131-175-156, fax: 49-6131-173-423, e-mail: kauczor@radiologie.klinik.uni-mainz.de, World Wide Web: http://www.uni-mainz.de/FB/Medizin/Radiologie/leshouches/Welcome.htm 